\title{
High Energy Neutron Transmission Analysis of Dry Cask Storage
}

\author{
Christopher Greulich*, Christopher Hughes, Yuan Gao, Andreas Enqvist, \\ James Baciak \\ Nuclear Engineering Program, University of Florida, 100 Rhines Hall, Gainesville, FL \\ 32611
}

\begin{abstract}
Since the U.S. currently only approves of storing used nuclear fuel in pools or dry casks, the demand for dry cask storage is on the rise due to the continuous operation of currently existing nuclear plants which are reaching or have reached the capacity of their used fuel pools. With the rising demand comes additional pressure to ensure the integrity of dry cask systems. Visual inspection is costly and man-power intensive, so alternative nondestructive testing techniques are desired to insure the continued safe and effective storage of fuel. One such approach being investigated by the University of Florida is neutron based computed tomography. Simulations in MCNP are preformed where D-T energy neutrons are transmitted through the dry cask and measured on the opposite side. If the transmitted signal is clear enough, the interior of the cask can be reconstructed from the measurement of the alterations of neutron signal intensity using standard mathematical techniques developed for medical imaging. Preliminary efforts show a correlation between energy and number of scatters (which is an indication of retention of position information). Work is ongoing to quantify if the correlation is strong enough that an energy discriminator may be used as a filter in future image reconstruction. The calculated transmission probability suggests that an image could be reconstructed with a week of scanning.
\end{abstract}

Keywords: Neutron Tomography, Used Nuclear Fuel, Dry Cask Storage, Imaging, Nondestructive Testing, Computed Tomography

\footnotetext{
*Corresponding author

Email address: cgreulich@ufl.edu (Christopher Greulich )
} 


\section{Introduction}

\subsection{Motivation}

The U.S. continues to operate nearly 100 existing nuclear reactors, of which their respective plants are reaching or have reached the capacity of their used

5 fuel pools which necessitates an alternative storage solution. Dry cask storage allows used fuel that has already been cooled in the used fuel pool for at least five years to be surrounded by an inert gas inside a specially designed and licensed cask; which, while expensive, is a fraction of the cost to construct an additional used fuel pool or develop fuel reprocessing infrastructure. Currently,

1075 of the 78 U.S. reactor sites either have or are pursuing an independent spent fuel storage installation (ISFSI) for dry cask fuel storage [1 with over 2,000 casks already loaded [2]. Cask loading, and the increasing need for continual testing, will only continue to grow until a final repository solution is operational or the U.S. begins to recycle fuel. The challenge of monitoring the condition of the used fuel inside the cask without exposing the used fuel has been evident since the beginning of dry cask storage in the 1980s, and especially when it became apparent that dry cask storage, which was initially designed for short term storage, may transition into longer term storage. The prevailing method for fuel monitoring is a robust safeguard regime that maintains continuity of knowledge, where the contents of a cask are loaded in the presence of inspectors and the casks are fixed with tamper indicating tags and seals that are than periodically inspected.

Were a tag or seal to indicate tampering, the cask would most likely have to be reopened given that no current accepted and verified methods exist to reestablish continuity of knowledge without visual inspection. Under current regulations and operational procedures, dry handling, even remote dry handling, of used fuel is not often performed. Used fuel is typically only manipulated while submerged fully underwater. Exelon reported that just to load a cask cost five hundred thousand dollars in addition to the one million dollars for a cask itself 3]. This makes visual inspection of the cask interior disadvantageous since the cost to move a cask back to the spent fuel pool, breach the seals, perform 
the inspection, re-dry and seal the cask, then move it back into position would probably require at least twice the cost to load and several man months of effort. A majority of the casks in use [2] feature a fully enclosed canister sealed with 35 a weld. This presents even more complications for visual inspection since, to date, no welded cannisters have been re-opened.

This work focuses on MCNP simulations where an external D-T neutron generator creates a source beam that was transmitted through the cask to an array of detectors which were placed on the opposite side of the cask. If the source and detectors are rotated completely around the cask, the planar radiation projection is generated, more commonly called the Radon transform. This projection can be processed with filtered back projection or other more advanced reconstructive imaging algorithms to recreate the interior structures. The mathematics of image reconstruction and the Radon transform date back 45 to 1917 when first introduced by Johann Radon. Modern computed tomography was invented in 1972 by Godfrey Hounsfield, who took the theoretical calculations of Allan Cormack and created the first CT scanner. CT scanning saw widespread adoption by the medical field in just a matter of years.

The imposed restrictions on conventional visual inspection gives other nondestructive analysis techniques considerable leeway in design parameters. Such systems could cost multiple millions of dollars if they were reusable and could measure several casks. Casks are initially licensed or certified for 20 years with possible renewals of an additional 40 years [4]; therefore measurements times of weeks to months, even possibly a year or more would be feasible assuming 55 the system was mostly automated and the required man-hours were considerably less than visual inspection. Additionally, less detailed resolution would be acceptable if the cost was appropriately scaled.

\subsection{Previous Work}

A majority of the historical work for used fuel has been on verification, diversion detection, and burn-up credit validation done mostly on the assembly level in order to meet regulatory guidelines. One of the first, more successful efforts at full cask measurement was done by a Idaho National Laboratory group. They developed the Compton Dry-Cask Imaging Scanner that has the ability to detect empty vs full storage positions but it suffered a setback when transported 
to the Doel Nuclear Power Plant near Antwerp, Belgium; the system was unable to detect full energy peaks because of the additional scattering caused by a thick ballistic steel shield [5].

As detectors, computational power, and modeling improves, a few groups have leveraged these advancements and started to investigate CT hoping, that despite the difficulties, the technique can give information on structural condition. The downsides of $\mathrm{CT}$ versus simpler verification and validation techniques are the amount of data required and the time it takes fully resolve useful images. Previous works have shown the basic technology can feature image resolutions from sub-centimeter to several centimeters depending on the design parameters. 75 Groups have mostly limited the tomographic work to a per assembly basis using gamma rays [6] 8 , rather than the full dry cask.

An Idaho National Laboratory (INL) group in 2005 utilized then current gamma and neutron imaging systems to measure six dry storage casks stored at INL [9]. They concluded that the low energy regimes they measured suffered from too much scattering to effectively differentiate casks, although they noted evidence of "significant fluences of higher-energy, unscattered radiation" [9]. Recently a group at Missouri University of Science and Technology completed a simulation study of high energy x-ray CT measuring the entire dry cask [10; they demonstrated that under ideal conditions sub-centimeter resolution could 85 be obtained with scanning times of $1000 \mathrm{hr}$ using a monoenergetic $4 \mathrm{MeV}$ photon source with a source strength of $1 \times 10^{15}$ photons/s [10].

Neutron tomography has not been as widely developed as gamma tomography due to the relative difficulty of detecting neutrons with high spatial resolution. Neutron CT has been mostly used as varied scientific applications such 9o as fossil study [11] and the imaging of water penetration in pretzel M\&M's [12]. A dissertation from 2010 entitled Spent Nuclear Fuel Assembly Inspection using Neutron Computed Tomography [13] showed that neutron tomography could be utilized on nuclear fuel assemblies. The work's utilization of ideal miniature simulated neutron detectors means significant practical engineering would be required to achieve any of the shown results. More recently, Eric Rauch of LANL conducted work on neutron fingerprinting [14, which in the simplistic analysis performs much the same measurements as computed tomography but 
stops short of the inverse transform; the work simulated 100 He-3 tubes placed around a dry cask which was loaded with varying assembly signatures. These signatures were modeled using an Oak Ridge National laboratory team's [15] best estimate used nuclear fuel source terms reconstructed from NRC documents. The work concluded that the unique arrangement of neutron sources, where assemblies had variable burn-ups, produced a "unique identification" of the cask that has been shown to be "durable over time" which could "restore continuity of knowledge" if "previous measurements were available for comparison" [14.

\subsection{Practical Considerations}

The major challenge Ziock et al. [9] identified for this type of tomography work was the impact of scattering on the low energy particles they were detecting. Tomography is primarily meant for unscattered particles since that gives a clear indication of previous particle locations; it is possible to tomographically reconstruct images based on particles that have undergone a single scatter, since their likely spatial history can be recomputed given kinematics and knowledge of the scattering medium. Unfolding particle track information for particles that have undergone multiple scatters is mathematically ambiguous and rarely preformed. Numerous conventional ways exist to cut down on the scattered signal. A method commonly used is an anti-scatter grid or collimator. A collimator is an additional layer of shielding placed between the object of interest and the detectors, which features holes or channels which should permit only radiation traveling perpendicular into the detector pixel; any radiation tracks incoming at an oblique angle would strike the side of the collimator wall before entering the detector and thus be absorbed in the collimator. This approach has merit but given the high attenuation of the cask it is hard to design a collimator that could effectively stop what the cask was only able to scatter. Were a collimator to effectively collimate the beam, the additional attenuation would be detrimental to the already significant measurement times.

Another approach is to perform background subtraction using a point just outside the edge of the unscattered cone and taking this measurement as the contribution of scatter along the entirety of the detector array. This method 
assumes a majority of the signal comes from unscattered events and the scattered background contributes a small amount. This assumption may not be valid in this context. Background subtraction could be achieved with a physical measurement or a simulation. This is problematic given the size of the detector array and the distance from the source. Due to the cone geometry of the source, the scatter contribution is expected to mostly mirror the semicircular distribution of the primary unscattered radiation, which was confirmed by results of preliminary simulations. The contribution near the center of the flat array should be higher than the contribution near the edges since the distance to the source is reduced. Additionally, the scatter contribution would be a signification fraction of the measured signal; thus the uncertainty of the remaining signal would be significant after correction. Futhermore, it was determined that due to vertical orientation of the fuel rods the beam would preferentially widen in the vertical direction and be attenuated in the horizontal direction. This would create different spatial image resolutions in the horizontal and vertical direction, which the background subtraction method would have to take into consideration adding further complexity to this method.

Due to the difficulties inherit to conventional methods of dealing with downscatter to cope with this application, a new simpler method is preferred. Scattering at its core is a method for neutrons to thermalize or lose energy. Quantifying the average energy of a neutron as a function of number of scatters is practical given a uniform medium of known dimensions. Given the material inhomogeneity and the several mean free paths between the detector and source, the quantification becomes difficult. A single hydrogen scatter in the resin shield can cause the neutron to lose all of its energy, while a single uranium-238 scatter can cause the neutron to lose, on average, less than $1 \%$ of its energy. Due to this disparity, the distribution of particle energy by number of scatters in the cask is most easily determined stochastically. Once the distribution is known, a threshold value could be determined such that neutrons above the energy threshold are likely to have undergone a lower number of relatively small angle scatters. If the number and angle of the scatters are low the events may retain enough position information to reconstruct an image. 


\section{Description of Model and Simulations}

A Transnuclear (TN) 32 cask (NRC certificate of compliance number 721021) was simulated and analyzed. The cask features a bolted lid design that stores 32 pressurized water reactor assemblies. Figure 1 shows two views of the MCNP [16] model. The model was based on a SolidWorks model provided by the vendor.

[Figure 1 about here.]

While Figure 1 shows the assemblies as homogenized for ease of viewing, the igh energy neutron is more likely to transmit through the cask, and the energy is well outside the normal fission source and natural background distributions, so interference should be minimal.

To ensure maximum geometric efficiency, the detector tally was a plane perpendicular to the source path immediately adjacent to the cask. The MCNP particle track output (PTRAC) option was used to track each particle that crossed the surface of the detection plane. The input file was replicated and ran on 343 individual threads for a total of 4 days creating roughly a million events 
in 7 billion neutron histories which was stored in 343 PTRAC files totaling 400 gigabytes. The PTRAC files were first parsed into a more human readable format with ParsNIP [17] then analyzed with a self developed MATLAB program.

\section{Results and Discussion}

An initial flux estimation using a super imposed mesh tally (fmesh) was used to identify how well neutrons transmitted through the cask. The fmesh was discretized over energy into two bins: low energy $(\leq 10 \mathrm{MeV})$ and high energy $(>10 \mathrm{MeV})$. Figure 3 shows the cask flux of the two different energy bins for a starting angle of $0^{\circ}$. Figure 4 shows the result of the fmesh tally for the two energy bins with an intial angle of $45^{\circ}$. The low energy bin or down scattered images of Figures 3(a) and 4(a) show the extent to which the cask broadens the cone beam and creates noise across the detector region, which would need to be filtered. Figure $3(\mathrm{~b})$ shows the potential for streaming terms, where the primary beam would not interact with any fuel which creates higher counts than observed when no streaming term exists like Figure 4(b). Combining the MCNP output and the four fmesh plots, information about the neutron transmission can be deduced. Neutrons scatter off the iron and helium and then are absorbed by boron, but an appreciable number of neutrons do transmit through the cask. This along with the streaming term introduces the possibility of image reconstruction. If nothing else the streaming term may be used with simple counting to demonstrate large positional shifts or reorganization of the fuel.

[Figure 4 about here.]

Figures 3 and 4 show a strong back scatter component, despite the declining probability of back scatter at high energies. The expected depth of penetration is very small, therefore the back scatter methodology may have trouble imaging the interior fuel structures. Future work may attempt to quantify the penetration depth.

Figures 5 shows the energy distribution of events that cross through the cask. The distribution shows how effective the cask is at moderating and shielding 
radiation. The average particle exits the cask at $0.31 \mathrm{MeV}$ after undergoing an average of 122 collisions. The number of events drops off rapidly with energy. Figures 5 shows two distributions one for all events that cross the far plane of the cask and one for just events within a narrow bounding box that represents the potential physical detector. Figure 6 shows the bounding box around the circular primary unscattered source cone. The energy distribution for the narrow region is similar to the total but slightly shifted towards higher energy. The population statistics for the two distributions are within the uncertainty of each other. Figure 6 shows the location of all events that cross completely through the cask and have scattered fewer than 10 times. Most events scatter completely outside of the cask, but a few transmit through the cask relatively undisturbed.

[Figure 6 about here.]

The energy distribution binned by number of scatters is shown in Figure 7

[Figure 7 about here.]

The first thing of note is the extremely small number of counts with fewer than 2 scatters, which represents the ideal number for image resonctrution since the particles retain the most spatial resolution information. There were no events that passed completely through the cask without interacting once, and only 9 counts that interacted only a single time before exiting the cask. This was due in large part to the high cross section of the borated polyester resin neutron shield. Alternative cannister type systems which typically replace the neutron shielding resin with a thick concrete overpack may allow for better transmission. As expected the energy distributions shifts lower in energy as the number of scatters increase. The average energies of events with fewer than 7 scatters remains relatively high which suggests that the neutrons undergo several small angle scatters which would allow for the possibility to utilize multiple scattering theory and reconstruct an image with an additional noise term compared to traditional image reconstruction.

A one-way analysis of variance (ANOVA) statistical test was performed on the various distributions. The p-value was zero to the working precision of 
MATLAB; This means we can reject the possibility that the distributions were simple random samplings from a common distribution. This low p-value was the result of the high number of samples in each distribution with the exception of the single scatter distribution which features very few events. While the ANOVA p-value gives clear indication that the separation of means of the distributions are statistically significant, it does little to quantify if the overlapping distributions can be properly resolved and separated when only the total energy distribution is given. To quantify the ability to separate the distributions, Figure 8 was created, which shows the sum of the events with fewer than the number of collisions stated and in the energy range given up to the maximum energy range divided by the sum of all events in the energy range given up to the maximum energy.

\section{[Figure 8 about here.]}

Roughly $80 \%$ of the signal above a few $\mathrm{MeV}$ comes from events that scatter fewer than 10 times despite the average particle scattering 122 times. The graph shows the expected trend of increasing fraction with energy. At number of average scatters is inversely proportional to the minimum energy. With no energy threshold the average event scatters $122 \pm 80$, but with a threshold of 11 $\mathrm{MeV}$ the average event scatters only $8 \pm 4$ times. Therefore it may be possible to implement an energy discriminator so that the recorded events are more likely to retain positional information.

In order to address the potential regulatory issues, an MCNP simulation was performed to quantify the fission reaction rate. Results showed that roughly for every 60 source neutrons only one resulted in a fission. When the reaction rate is combined with the beam intensity of the best case neutron generator and the average energy per fission the result is the heat generation rate. The product comes to an additional volumetric heat generation rate on the order of microwatts per assembly. The additional heat rate is well within the margin of error for the designed dissipation rate of 1.02 kilowatt per assembly [18].

Increased dose is another regulatory concern. Results show that roughly $80 \%$ of the neutron beam is absorbed by the cask. The source intensity of the beam is roughly equivalent to the emissions from a single cask but with a higher average energy therefore the neutron radiation weighting factor would be 
lower. The combination of the lower radiation weighting factor and absorption by the cask results in a lower dose than that from a single cask. An ISFSI typically has several casks present, therefore the additional dose of the scanning should be negligible. With a perfect detector array 100 centimeters wide by 150 centimeters tall roughly $1 \times 10^{-7}$ events per starting particle are detected with remaining energies above $11 \mathrm{MeV}$. Assuming a neutron generator that generate $10^{8}$ neutrons a second, a measurement time on the order of a week or more would be required to ensure measurements of a few thousand counts in each pixel of a detector consisting of a few hundred pixels. Newer neutron generators are in development that offer substantially higher flux which will reduce the projected required time.

\section{Conclusions}

Alternatives to visual inspection of dry cask storage are desired to ensure the continued safe and effective storage of fuel, and to recertify casks if continuity of knowledge is ever lost. Neutron imaging is a promising method. A major issue with imaging casks is the amount of scattering in the cask which degrades image resolution. A modification to conventional imaging is required since traditional imaging techniques employ unscattered particles which are extremely unlikely to survive through the entire cask. A TN-32 dry storage cask was modeled in MCNP with an impinging $14.1 \mathrm{MeV}$ mono-energetic $8^{\circ}$ conical neutron source near the side of the cask. All events that crossed the plane on the opposite side of the cask were recorded. The simulations show a correlation between remaining energy of a neutron transmitted through a cask and number of scatters. With no energy threshold the average event scatters $122 \pm 80$, but with a threshold of $11 \mathrm{MeV}$ the average event scatters only $8 \pm 4$ times. With the energy threshold, multiple scattering theory could be applied since it is likely that events with low number of scatters underwent mainly small angle scatters; therefore there is a possibility of image reconstruction if the noise of the multiple scattering is properly corrected. To insure proper detector statistics for image reconstruction of a dry cask, a scanning time on the order of a week or more would be required. 


\section{Acknowledgments}

This material is based upon work supported in part under an Integrated University Program Graduate Fellowship. Additionally, this publication was made possible through support provided by the U.S. Department of Energy under the terms of Agreement No. DE-NE0008400. The opinions expressed herein are those of the author(s) and do not necessarily reflect the views of the U.S. Department of Energy. Finally, the University of Florida's Research 5 Computing and their HiPerGator high-performance cluster provided the access to computational resources.

\section{References}

[1] NRC, U.s. independent spent fuel storage installations (2015). URL http://pbadupws.nrc.gov/docs/ML1524/ML15240A058.pdf

[2] J. Carter, Consolidated interim storage facility design concepts in the u.s. (2015).

[3] M. L. Wald, A safer nuclear crypt, The New York Times. URL http://www.nytimes.com/2011/07/06/business/ energy-environment/06cask.html

[4] P. Rinard, G. Bosler, Safeguarding lwr spent fuel with the fork detector, Tech. rep., LA-11096-MS, Los Alamos (1988).

[5] J. Wharton, E. Seabury, G. Caffrey, et al., Summary report: Inl cdcis cask scanner testing at, Tech. rep., Idaho National Laboratory (INL) (2013).

[6] F. Lévai, S. Dési, M. Tarvainen, R. Arlt, Use of high energy gamma emission tomography for partial defect verification of spent fuel assemblies, Tech. rep., Finnish Centre for Radiation and Nuclear Safety (STUK) (1993).

[7] S. Jacobsson, A. Bäcklin, A. Håkansson, P. Jansson, A tomographic method for experimental verification of the integrity of spent nuclear fuel, Applied Radiation and Isotopes 53 (4) (2000) 681-689.

345 [8] S. J. Svärd, S. Holcombe, S. Grape, Applicability of a set of tomographic reconstruction algorithms for quantitative spect on irradiated nuclear fuel 
assemblies, Nuclear Instruments and Methods in Physics Research Section A: Accelerators, Spectrometers, Detectors and Associated Equipment 783 (2015) 128-141.

[9] K.-P. Ziock, G. Caffrey, A. Lebrun, L. Forman, P. Vanier, J. Wharton, Radiation imaging of dry storage casks for spent nuclear fuel, in: Conference record of IEEE Nuclear Science Symposium, 2005.

[10] X. Liu, H. K. Lee, A simulation study of the spent nuclear fuel cask condition evaluation using high energy x-ray computed tomography, NDT \& E International 80 (2016) 58-64.

[11] M. D. Sutton, Tomographic techniques for the study of exceptionally preserved fossils, Proceedings of the Royal Society of London B: Biological Sciences 275 (1643) (2008) 1587-1593.

[12] F. Munger, Weirdly wonderful: Ornl reactor's role sometimes veers from the norm, Knoxville News Sentinel.

URL http: //www . knoxnews. com/news/local/ weirdly-wonderful-ornl-reactors-role-sometimes-veers-from-the-norm-ep-360458413-35689 html

[13] C. L. Pope, Spent nuclear fuel assembly inspection using neutron computed tomography, Ph.D. thesis, Idaho State University (2010).

11 URL http://libpublic3.library . isu . edu/login?url=http: //search.ebscohost. com/login . aspx?direct=true\&db=cat03172a\& $\mathrm{AN}=$ isu $.1082614 \&$ site $=$ eds - live

[14] E. B. Rauch, Signatures of Extended Storage of Used Nuclear Fuel Comprehensive Final Report, 2016. doi:10.2172/1327981. URL http://www.osti.gov/scitech/servlets/purl/1327981

[15] J. Scaglione, J. Peterson, K. Banerjee, K. Robb, R. LeFebvre, Integrated data and analysis system for commercial used nuclear fuel safety assessments-14657, in: WM2014 Conference, 2014.

[16] T. Goorley, M. James, T. Booth, F. Brown, J. Bull, L. Cox, J. Durkee, J. Elson, M. Fensin, R. Forster, et al., Initial mcnp6 release overview, Nuclear Technology 180 (3) (2012) 298-315. 
[17] T. Hutton, Parsnip:parser for ptrac files produced by mcnp (2014). URL https://sourceforge.net/projects/ptracparser/

380 [18] Transuclear, Inc., Tn-32 dry storage cask system: Safety evaluation report Tech. rep., revision 11a (1999).

URL https://www.nrc.gov/docs/ML0036/ML003696918.pdf 


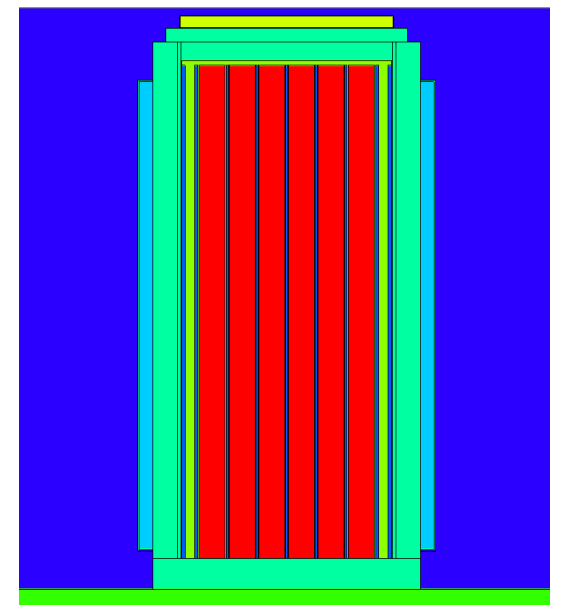

(a) XY Plot

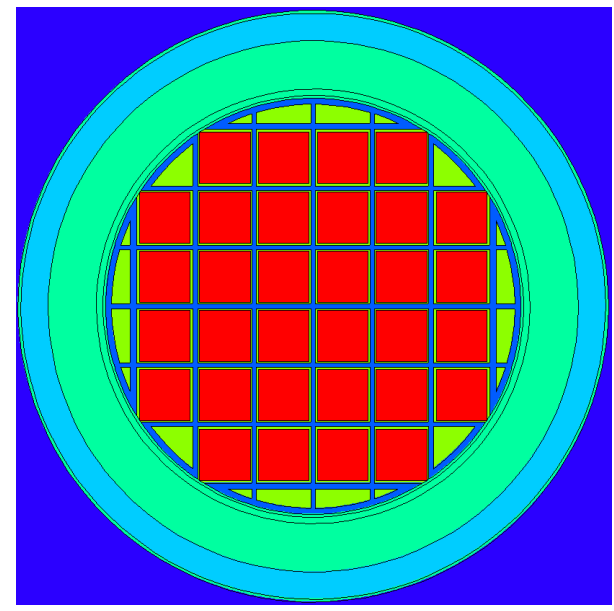

(b) XZ Plot

Figure 1: The visualization of a TN-32 cask MCNP model using VISED with the assemblies homogenized for ease of viewing. 


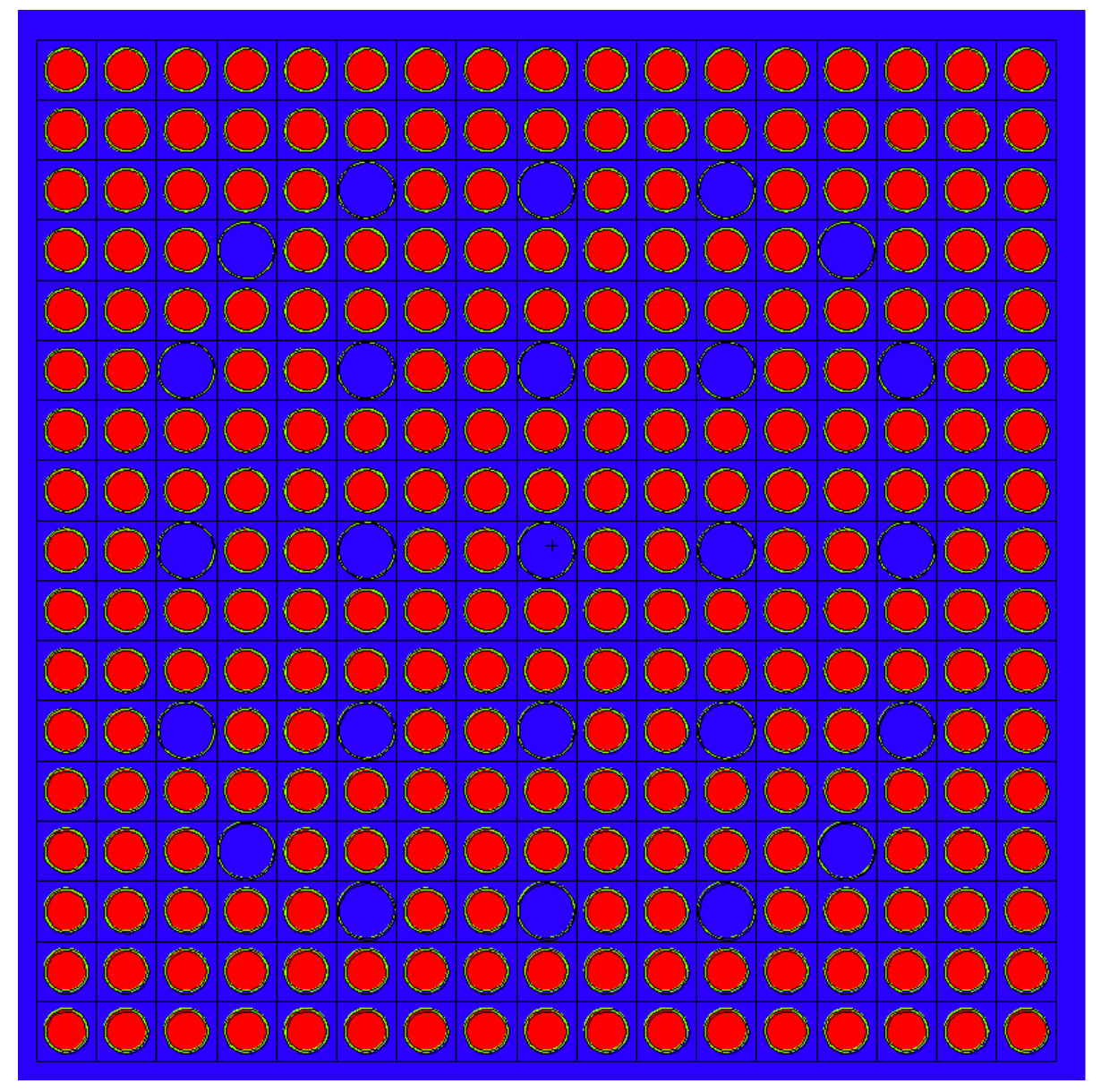

Figure 2: A cross-sectional view of the modeled 17x17 Westinghoud PWR fuel assembly.Note what may appear to be 15 empty spaces are actually instrumentation and guide tubes with very thin cladding. 


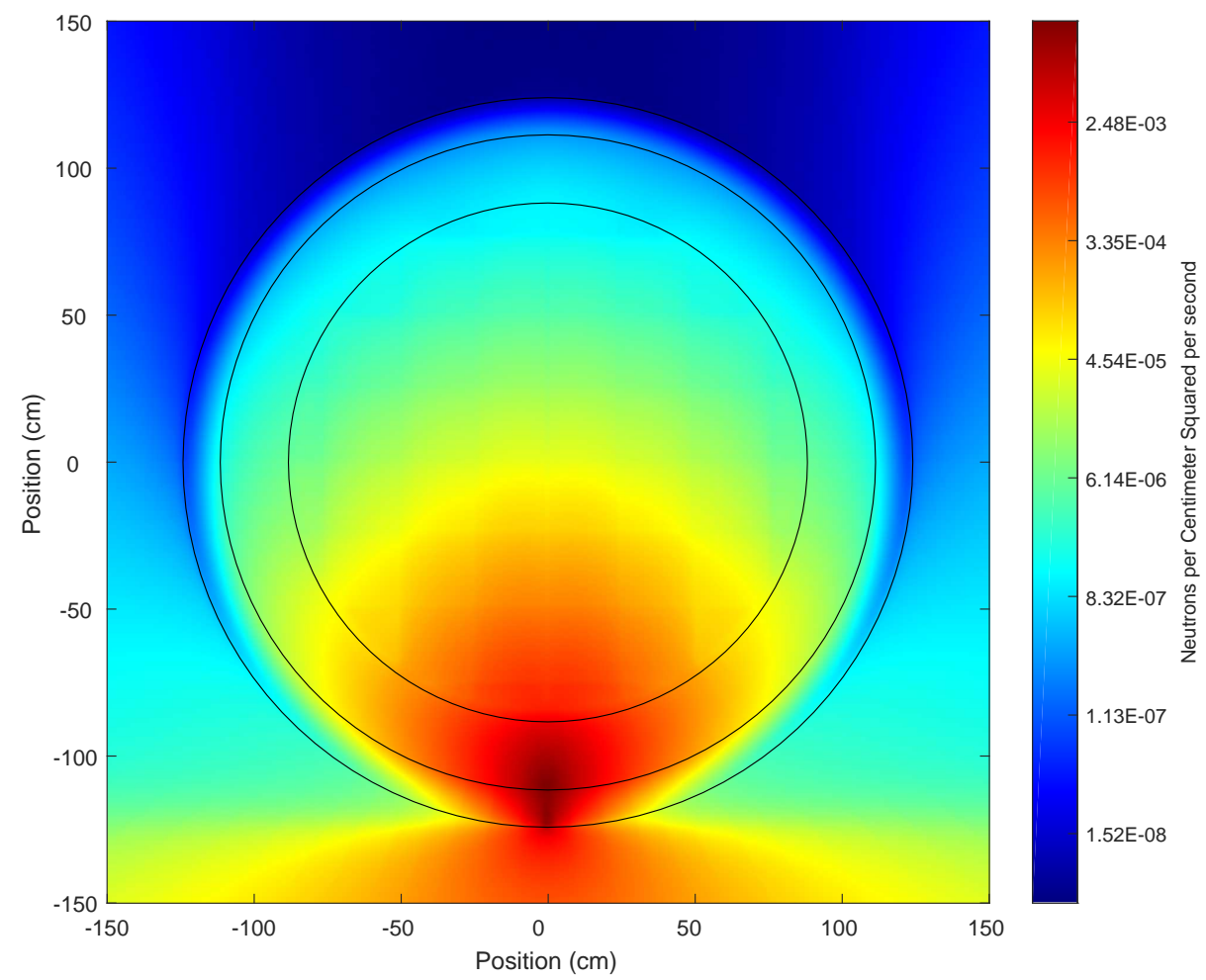

(a) 0 Degree Incident Angle - Low Energy

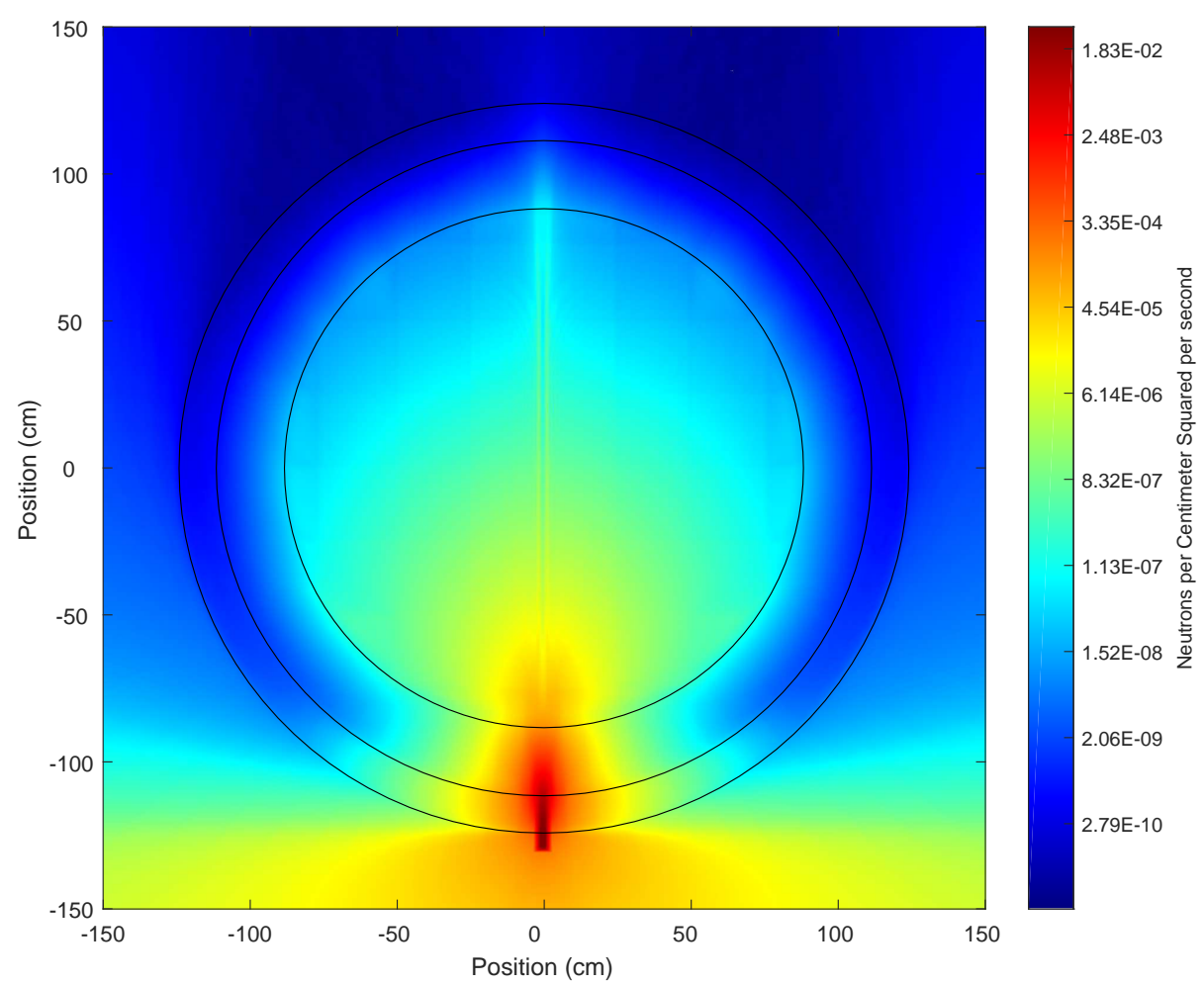

(b) 0 Degree Incident Angle - High Energy

Figure 3: The superimposed fmesh tally showing the flux distribution in neutrons per centimeter squared per starting particle for neutron energies in excess of $10 \mathrm{MeV}$. 


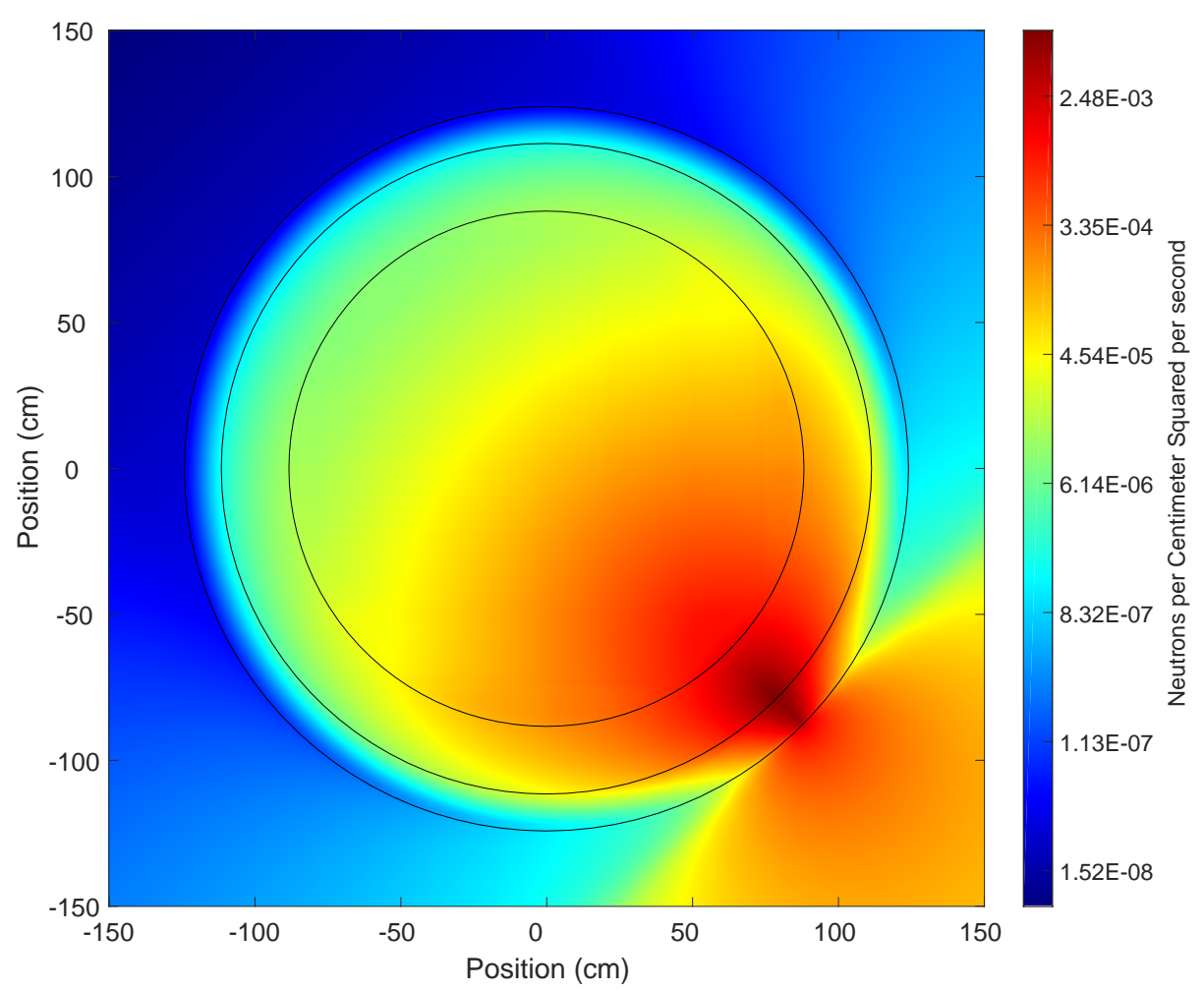

(a) 45 Degree Incident Angle - Low Energy

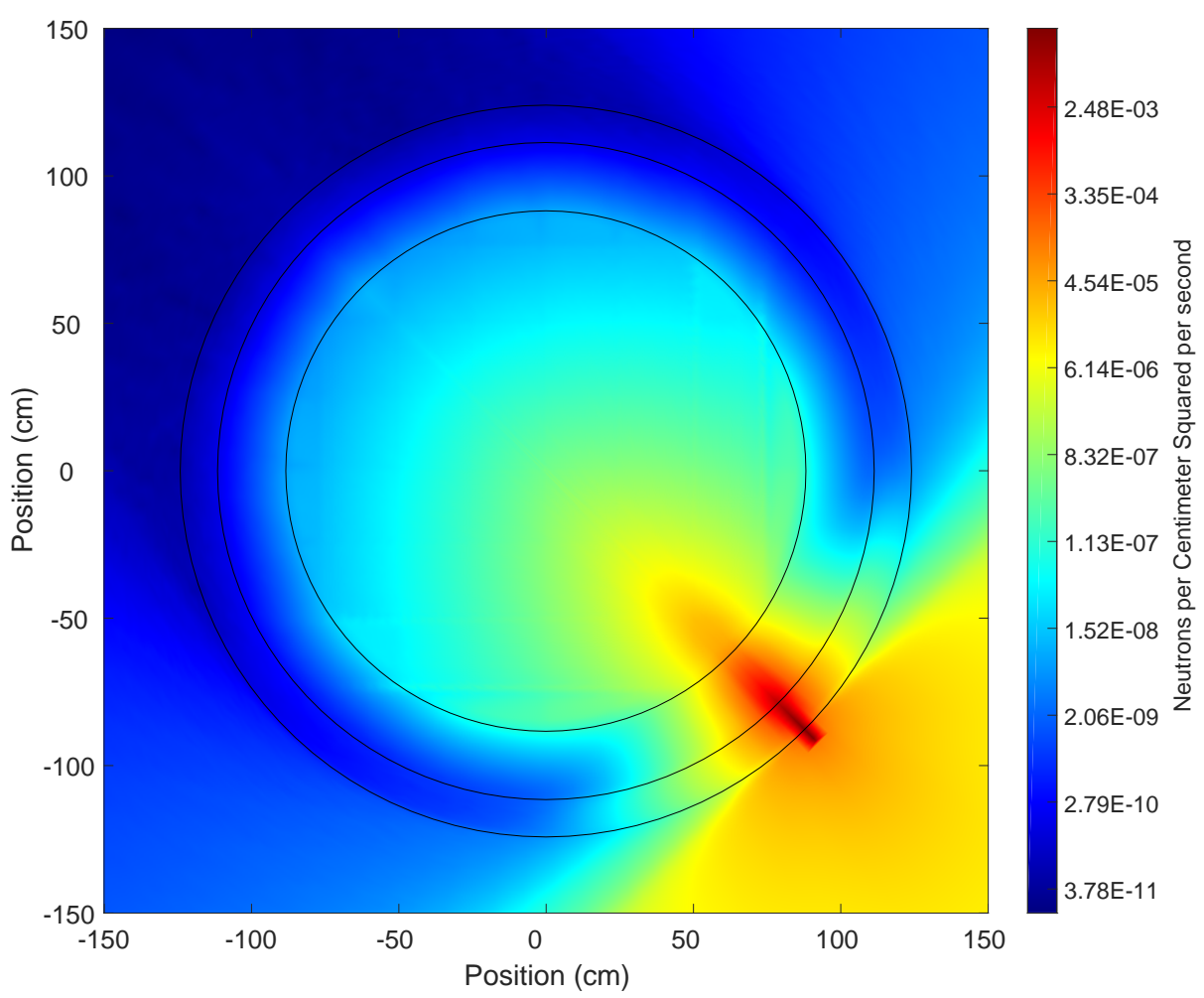

(b) 45 Degree Incident Angle - High Energy

Figure 4: The superimposed $\&$ mesh tally showing the flux distribution in neutrons per centimeter squared per starting particle for neutron energies below $10 \mathrm{MeV}$. 


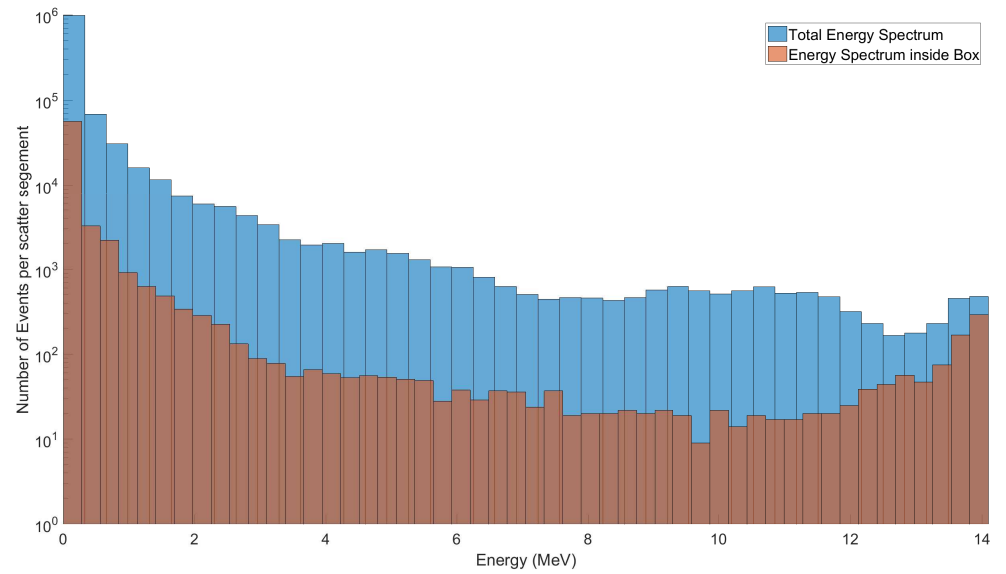

Figure 5: The energy distribution of all events that cross through the cask, and the distribution for events inside the bounding box shown in Figure 6 


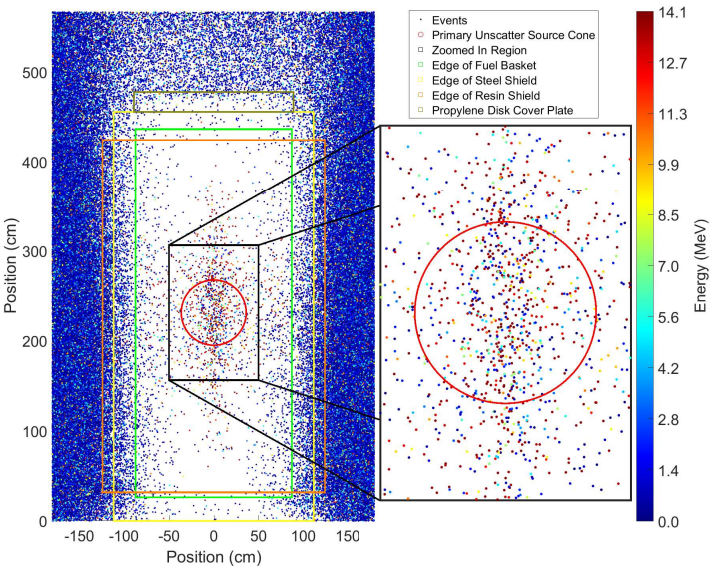

Figure 6: The plotted events with fewer than 10 scatters colored by energy. 


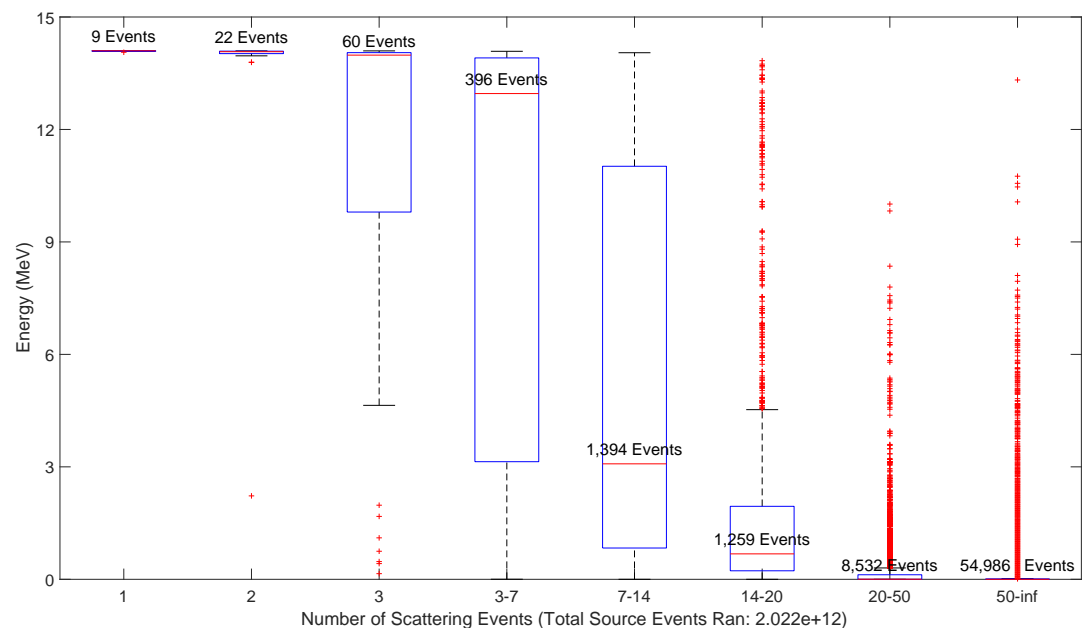

Figure 7: The energy distributions as a function of number of scatters. Each box plot shows the distribution of energy of events that scatter exactly the number stated or the inclusive range shown. 


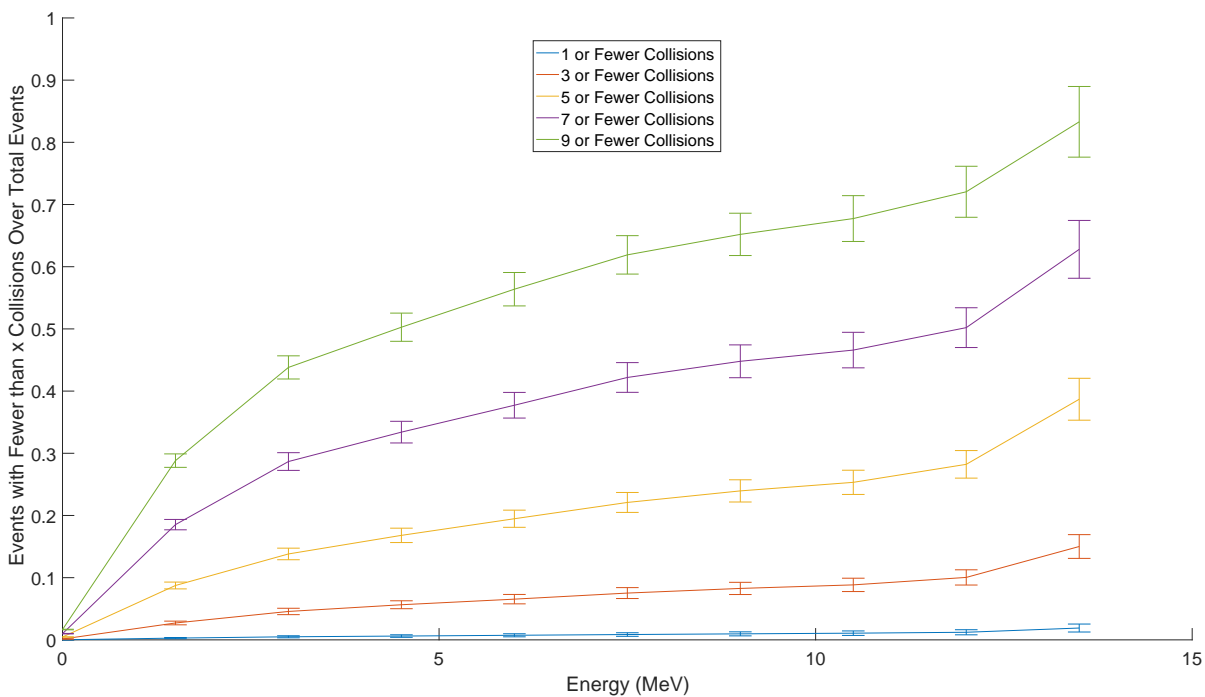

Figure 8: The fraction of events with fewer than the stated number of collisions over the total number of events for the range of the given energy to the maximum energy. 